Behandlungsfalls zu einer neuerlichen eingehenden Beratung, ist das eine Begründung in sich selbst. Das kann durchaus genau so formuliert werden: "Erkrankung hat neuerliche Beratung erforderlich gemacht." Nicht vergessen sollte man, dass auch eine telefonische Beratung zum Ansatz der Nr. 3 berechtigt. Und selbst eine indirekte Beratung über eine berechtigte Bezugsperson, etwa
Familienangehörige und Pflegekräfte, ist nicht ausgeschlossen.

Aufpassen heißt es bei der mitunter nicht vermeidbaren Kombination einer Beratung, die die Kriterien der Nr. 3 erfüllt, mit sogenannten technischen Leistungen. Hier sollte man die jeweils höherwertige Abrechnungsvariante wählen und/oder die Möglichkeit einer Steigerung in Betracht zieht. Eine Ergo- metrie z. B. ist mit 59,66 Euro (2,3-facher Steigerungssatz) deutlich höher bewertet als die Nr. 3 mit 20,10 Euro (2,3-facher Steigerungssatz). Die Nr. 3 muss also wegfallen, um die Ergometrie nicht auszuschließen. Um die Beratung trotzdem noch zu erfassen, bietet sich als Ersatz die Nr. 1 an, ggf. mit einem höheren Multiplikator und der Begründung "zeitlich aufwändige Beratung" (Tab. 1).

\title{
Heilmittelverordnung bei bestimmten Lymphödemen künftig regressfrei
}

_Zwei aktuelle Beschlüsse des Gemeinsamen Bundesausschusses (G-BA) wirken sich auf die Heilmittelversorgung aus. Zum einen wird die ambulante Ernährungstherapie für seltene angeborene Stoffwechselerkrankungen und Mukoviszidose zum 1. Januar 2018 als neues Heilmittel eingeführt. Darüber hinaus hat der G-BA die Diagnoseliste zum langfristigen Heilmittelbedarf im

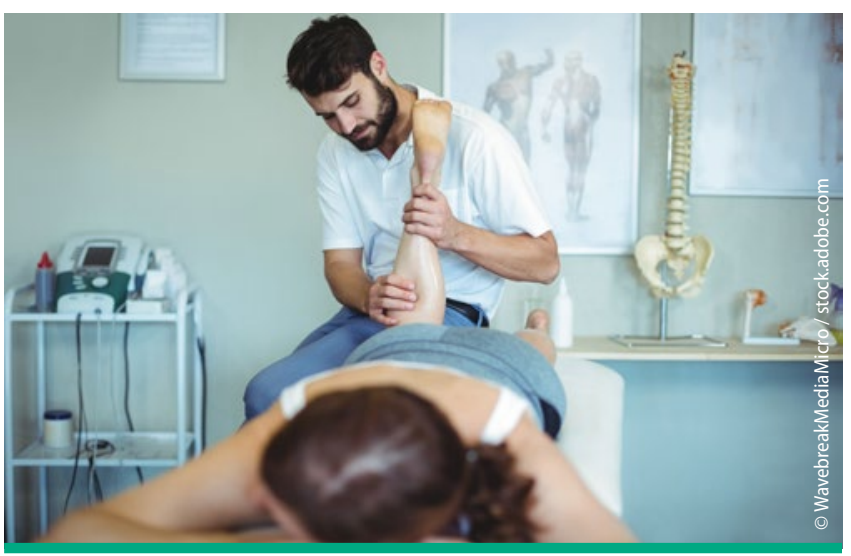

Tab. 2 Bei diesen Diagnosen ist eine Heilmittelverordnung ohne Regressangst möglich

Spezifikation des Lymphödems, ICD-10-Codes jeweils Stadium II oder III

allgemein I89.01, I89.02, I89.04, I89.05

nach (partieller) Mastektomie $197.21,197.22$

(mit Lymphadenektomie)

nach medizinischer Maßnahme am axillären oder inguinalen

197.82, 197.83, 197.85, 197.86 Lymphabflussgebiet

hereditär Q82.01, Q82.02, Q82.04, Q82.05
Bereich der Lymphödeme angepasst. Verordnungen von manueller Lymphdrainage für Lymphödeme des Stadiums II und III unterliegen dadurch nicht mehr der Wirtschaftlichkeitsprüfung und können ohne Regressangst verordnet werden.

Einige Lymphödeme waren bereits Anfang des Jahres vorübergehend als Diagnosen mit besonderem Versorgungsbedarf anerkannt worden (siehe MMW 1/2017, S. 23). Der neue G-BA-Beschluss schafft nun eine solide Basis.

\section{MMW-KOMMENTAR}

Im Rahmen der jährlichen Aktualisierung der ICD-10GM durch das Deutsche Institut für Medizinische Dokumentation und Information wurde die Kodierung der Lymphödeme in Stadien und Lokalisation aufgegliedert. Im Zuge dieser Überarbeitung haben medizinische Fachgesellschaften darauf hingewiesen, dass Lymphödeme bereits ab dem Stadium II langfristig mit Heilmitteln behandelt werden müssen - und deshalb die Diagnoseliste zum langfristigen Heilmittelbedarfergänzt werden müsse. Nach dem Beschluss kann man bei der Verordnung für viele Patienten mit Lymphödemen (Tab. 2) einen langfristigen Heilmittelbedarf nach Anlage 2 der Heilmittel-Richtlinie unterstellen. Das Bundesgesundheitsministerium muss den Beschluss noch prüfen, wofür es zwei Monate Zeit hat. Danach wird der Beschluss im Bundesanzeiger veröffentlicht und tritt in Kraft.

Der G-BA hat außerdem beschlossen, dass der therapeutische Nutzen und die medizinische Notwendigkeit für eine Ernährungstherapie für seltene angeborene Stoffwechselerkrankungen belegt ist. Dazu zählen die Phenylketonurie (PKU), Harnstoffzyklusdefekte und Formen von Glykogenose und Mukoviszidose. Für diese Krankheiten hat der G-BA die Ernährungstherapie als alternativlose medizinische Maßnahme eingestuft, da ohne sie Tod oder schwere Behinderung drohen. Künftig bezahlen die Krankenkassen deshalb eine ernährungstherapeutische Behandlung durch Fachkräfte wie Diätassistenten, Ökotrophologen und Ernährungswissenschaftler. Verordnen können die Behandlung allerdings nur Vertragsärzte, die auf die Behandlung von seltenen angeborenen Stoffwechselerkrankungen oder Mukoviszidose spezialisiert sind. Nur unter bestimmten Voraussetzungen könnten auch z. B. Hausärzte in Abstimmung mit dem Spezialisten eine Folgeverordnung ausstellen. Ein Grund wäre beispielsweise, dass der Patient ein weit entferntes Zentrum aufsuchen müsste, bloß um das Rezept abzuholen.

Dr. Gerd W. Zimmermann, Hofheim 